Louisiana State University

LSU Digital Commons

$4-27-2010$

\title{
Water equivalent thickness values of materials used in beams of protons, helium, carbon and iron ions
}

\author{
Rui Zhang \\ University of Texas Graduate School of Biomedical Sciences at Houston \\ Phillip J. Taddei \\ University of Texas MD Anderson Cancer Center \\ Markus M. Fitzek \\ Midwest Proton Radiotherapy Institute \\ Wayne D. Newhauser \\ University of Texas Graduate School of Biomedical Sciences at Houston
}

Follow this and additional works at: https://digitalcommons.Isu.edu/physics_astronomy_pubs

\section{Recommended Citation}

Zhang, R., Taddei, P., Fitzek, M., \& Newhauser, W. (2010). Water equivalent thickness values of materials used in beams of protons, helium, carbon and iron ions. Physics in Medicine and Biology, 55 (9), 2481-2493. https://doi.org/10.1088/0031-9155/55/9/004

This Article is brought to you for free and open access by the Department of Physics \& Astronomy at LSU Digital Commons. It has been accepted for inclusion in Faculty Publications by an authorized administrator of LSU Digital Commons. For more information, please contact ir@lsu.edu. 


\title{
Water equivalent thickness values of materials used in beams of protons, helium, carbon and iron ions
}

\author{
Rui Zhang ${ }^{1,2}$, Phillip J Taddei ${ }^{2}$, Markus M Fitzek ${ }^{3,4}$, and Wayne D Newhauser ${ }^{1,2,5}$ \\ ${ }^{1}$ Graduate School of Biomedical Sciences, The University of Texas at Houston, 6767 Bertner, \\ Houston, TX 77030, USA \\ 2Department of Radiation Physics, Unit 1202, The University of Texas MD Anderson Cancer Center, \\ 1515 Holcombe Boulevard, Houston, TX 77030, USA \\ ${ }^{3}$ Midwest Proton Radiotherapy Institute, 2425 Milo B Sampson Lane, Bloomington, IN 47408, USA \\ ${ }^{4}$ Indiana University School of Medicine, 535 Barnhill Drive, RT 041, Indianapolis, IN 46202, USA
}

\begin{abstract}
Heavy charged particle beam radiotherapy for cancer is of increasing interest because it delivers a highly conformal radiation dose to the target volume. Accurate knowledge of the range of a heavy charged particle beam after it penetrates a patient's body or other materials in the beam line is very important and is usually stated in terms of the water equivalent thickness (WET). However, methods of calculating WET for heavy charged particle beams are lacking. Our objective was to test several simple analytical formulas previously developed for proton beams for their ability to calculate WET values for materials exposed to beams of protons, helium, carbon and iron ions. Experimentally measured heavy charged particle beam ranges and WET values from an iterative numerical method were compared with the WET values calculated by the analytical formulas. Inmost cases, the deviations were within $1 \mathrm{~mm}$. We conclude that the analytical formulas originally developed for proton beams can also be used to calculate WET values for helium, carbon and iron ion beams with good accuracy.
\end{abstract}

\section{Introduction}

Interest in heavy charged particle beam therapy has gradually increased since Wilson (1946) first proposed using particle beams in radiation therapy; he pointed out that the properties of specific ionization of heavy charged particles could be used for medical and biological applications. Since then, heavy charged particle beams used in radiotherapy have included protons and heavier ions such as carbon ions (Goitein et al 2002, Jakel et al 2003, Amaldi and Kraft 2005, Schulz-Ertner et al 2006, Schulz-Ertner and Tsujii 2007). The most important advantage of heavy charged particles is that they deposit most of their energy within a narrow range represented by the Bragg peak; this predictable deposition of dose can be utilized to spare normal tissues and kill malignant cells. In addition, the energies of heavy charged particle beams can be adjusted using modulating materials to treat any part of tumors within a patient.

During quality assurance testing with phantoms and radiotherapy treatment planning, liquid or solid water is frequently used to represent patient tissue (either by itself or in combination with other materials) for beam range and absorbed dose measurements, and water equivalent

(C) 2010 Institute of Physics and Engineering in Medicine

${ }^{5}$ Author to whom any correspondence should be addressed. wnewhaus@mdanderson.org. 
thickness (WET) or water equivalent ratio (WER) is often used to characterize the beam penetration range. For highly conformal heavy charged particle beam therapy with millimeter accuracy, it is very important to determine precise values of WET for each material in the beam line. Methods for calculating WET in proton beams (IAEA 2000, Newhauser 2001a, Newhauser et al 2007a) and heavy ion beams (IAEA 2000) were proposed previously, but these calculations required either a time-consuming iterative numerical method (NM) or fast but approximate methods of unknown accuracy. We recently derived several analytical formulas for this purpose and showed that these formulas can be used to calculate WET values for materials used in proton therapy of arbitrary density, elemental composition and thickness with small errors (Zhang and Newhauser 2009).

The objectives of this study therefore were to calculate the WET of various materials exposed to beams of protons, helium, carbon and iron ions using the formulas derived in our previous work and to compare our calculations to measured data. The analytical formulas were developed using theoretical range-energy relations, with the goal of achieving $1 \mathrm{~mm}$ uncertainty in WET.

\section{Methods and materials}

\subsection{Calculation methods}

The general formula to calculate WET for heavy charged particle beams is

$$
\mathrm{WET}=t_{w}=t_{m} \frac{\rho_{m}}{\rho_{w}} \frac{\bar{S}_{m}}{\bar{S}_{w}},
$$

where $t_{w}$ and $t_{m}$ are the thicknesses of water and the target material, respectively; $\rho_{w}$ and $\rho_{m}$ are the mass densities of water and the material, respectively; and $S_{w}$ and $S_{m}$ are the mean values of mass stopping power for water and the material, respectively (Newhauser 2001a). In essence, WET is the thickness of water that causes an ion beam to lose the same amount of energy as the beam would lose in some medium $m$. The unitless quantity WER is the ratio of $t_{w}$ to $t_{m}$. The methods for calculating the $\bar{S}$ value were extensively described in our previous paper (Zhang and Newhauser 2009) and are explained briefly here for the convenience of the reader.

The objective of each of the following three approaches was to calculate the mean mass stopping power in the material of interest based on relationships between theoretical mass stopping power and beam energy. The first relationship is based on the Bragg-Kleeman (BK) rule (Bragg and Kleeman 1905):

$$
S=-\frac{\mathrm{d} E}{\rho \mathrm{d} x} \approx-\frac{E^{1-p}}{\rho \alpha p},
$$

where $\alpha$ and $p$ are material-dependent constants and $E$ is the initial energy of the heavy charged particle beam. The values of $\alpha$ and $p$ were obtained by fitting to either residual ranges or stopping powers data (Ziegler et al 1985). A second range-energy relationship for heavy charged particle beams is captured in the Bethe-Bloch (BB) equation (Bethe 1930, Bloch 1933):

$$
S=-\frac{\mathrm{d} E}{\rho \mathrm{d} x}=4 \pi N_{\mathrm{A}} r_{e}^{2} m_{e} c^{2} z^{2} \frac{Z}{A} \frac{1}{\beta^{2}}\left[\ln \frac{2 m_{e} c^{2} \gamma^{2} \beta^{2}}{I}-\beta^{2}\right],
$$


where $N_{\mathrm{A}}$ is Avogadro's number, $r_{e}$ is the classical electron radius, $m_{e}$ is the mass of an electron, $z$ is the charge of projectile, $Z$ is the atomic number of the absorbing material, $A$ is the atomic weight of the absorbing material, $c$ is the speed of light, $\beta=v / c$, where $v$ is the velocity of the projectile, $\gamma=\left(1-\beta^{2}\right)^{-1 / 2}$ and $I$ is the mean excitation energy of the absorbing material. For compounds and mixture materials, the mass fractions of each constituent element are needed to calculate the effective atomic number $Z_{\text {eff }}$, the effective atomic weight $A_{\text {eff }}$ and the effective mean excitation energy, $I_{\mathrm{eff}}$, for the mixture as

$$
\begin{gathered}
Z_{\mathrm{eff}}=\sum a_{i} Z_{i}, \\
A_{\mathrm{eff}}=\sum a_{i} A_{i}, \\
\ln \left(I_{\mathrm{eff}}\right)=\frac{\sum_{i} a_{i} Z_{i} \ln \left(I_{i}\right)}{\sum_{i} a_{i} Z_{i}},
\end{gathered}
$$

respectively, where $a_{i}, Z_{i}$, and $I_{i}$ are the relative number of moles of element $i$ per mole of compound, the atomic number and the mean excitation energy of the $i$ th element in the compound, respectively (Leo 1987).

We previously proposed an empirical formula to calculate $I$ in an empirical form of the BB equation (EBB) (Zhang and Newhauser 2009), such that $I=k Z$, where $K=14.5$ when $Z \leq 8$, $K=13$ when $8<\mathrm{Z} \leq 13$ and $K=11$ when $\mathrm{Z}>13$.

An iterative NM of WET calculation described by Newhauser et al (2007a) was used as one of the standard of comparison for the other analytical formulas used here, because it is the most exact one of the methods considered.

For 'radiologically thick' targets (following the definitions of 'radiologically thick' or 'thin' from Zhang and Newhauser (2009)), changes in the projectile energy and mass stopping power must be considered. Therefore, we used $\bar{S}$ instead of point values $S$ in such cases (the 'thicktarget approach'). The $\bar{S}$ values were calculated by integrating equations (2) and (3) over projectile energy, where the limits of integration were the projectile energy at the entrance and exit of the slab. The $\bar{S}$ values were substituted into equation (1) to obtain WET values for thick targets. For 'radiologically thin' targets, one may use point value stopping powers established in equation (1) (the 'thin-target approach'). However, in this report we included only the thicktarget approach for brevity and because it is more robust and accurate than the thin-target approach.

\subsection{Measuring the WET for various materials in heavy charged beams}

Proton beam ranges were measured at the Midwest Proton Radiotherapy Institute (MPRI; Bloomington, IN). The MPRI houses a fixed horizontal beam line with a double scattering nozzle and two rotating gantries utilizing uniform scanning nozzles. It was built by the Indiana University Cyclotron Facility which also provides a cyclotron for acceleration of protons to a maximum energy of $208 \mathrm{MeV}$ (Anferov et al 2006). All proton experiments described here were performed in the fixed horizontal beam line (figure 1(a)). The double scattering system 
includes two scattering foils to spread and flatten the beam. The first foil is made of lead or a combination of lead and polycarbonate, depending upon the beam energy selection. The second foil is made of lead and lucite. Dose monitoring chambers are used to monitor and adjust the proton beam. Downstream of the scattering foils and the second dose monitoring chamber the beam passes through a range modulator and the snout with a final collimator.

A parallel-plate ionization chamber (Markus, model 23343, serial no 3997, PTW, Freiburg, Germany) coupled with an electrometer (Wellhofer Dosimetrie, model WP5007, serial no 3245 , Nürnberg, Germany) was used to measure the distribution of percentage of dose versus depth using a one-dimensional scanning water phantom system (IBA, model 2001, serial no 4739, Scanditronix Wellhofer, Bartlett, TN, USA). The beam range was taken as the distal depth along the beam central axis in water corresponding to $90 \%$ of the maximum dose $\left(D_{\max }\right)$. First we measured the range in water without the material of interest in the beam line $\left(R_{1}\right)$; then, we measured the range in water with the material placed immediately upstream of the water tank $\left(R_{2}\right)$. WET was the difference of these two ranges (i.e., $\left.R_{1}-R_{2}\right)$. Figure $1(\mathrm{~b})$ shows the experimental apparatus at the MPRI, including the water tank and a slab of interest.

Similar measurements were performed in passively scattered beams at the Northeast Proton Therapy Center (NPTC, Boston, MA). That system was described elsewhere (Jongen et al 1996). The WET values were deduced from range measurements made with a parallel-plate ionization chamber (Markus, model 23343, serial no 2397, PTW, Freiburg, Germany) that was used with a commercial one-dimensional scanning dosimetry system (Computerized Radiation Scanners, model 140, serial no 311, Vero Beach, FL, USA), which included an electrometer that was modified for proton therapy applications (Newhauser 2001a, 2001b). The ionization charge measurements and methods for determination of the proton range were described previously (Newhauser et al 2002).

Table 1 lists the materials used in this study, including gold, lead, stainless steel, titanium, aluminum, polycarbonate resin (Lexan, $\mathrm{C}_{16} \mathrm{H}_{14} \mathrm{O}_{3}$, GE Plastics Inc., Pittsfield, MA), polymethylmethacrylate (PMMA) (Lucite, $\mathrm{C}_{5} \mathrm{H}_{8} \mathrm{O}_{2}$, GE Plastics Inc., Pittsfield, MA), lung substitute plastic (LN300, Gammex RMI, Middleton, WI, USA), polystyrene, high-density polyethylene (HDPE), polyvinylchloride (PVC) and bone substitute plastic (SB3, Gammex RMI, Middleton, WI, USA). Also listed are their mass densities $\rho,(Z / A)_{\text {eff }}$ values (ratio of $Z_{\text {eff }}$ to $A_{\text {eff }}$ ) and mole fractions. The set of target materials included a wide variety of mass densities and was representative of materials commonly encountered in charged particle radiotherapy.

We tested whether the proton beam field size would affect the beam range results by using different size collimating apertures (with diameters of 10,8 and $5 \mathrm{~cm}$ ) with a $200 \mathrm{MeV}$ proton beam and with a 10 by $10 \mathrm{~cm}^{2}$ aluminum slab present. These measurements were carried out at MPRI.

Moyers et al (2009) previously measured the WER of different materials used in heavier charged particle beams of various energies at Loma Linda University and the National Aeronautics and Space Administration (NASA) Space Radiation Laboratory at Brookhaven National Laboratory (Upton, NY). These measurements, which were conceptually similar to the range measurements described above, were used in this work to confirm the values predicted using analytical formulas.

\section{Results}

Table 2 lists $\alpha$ and $p$ values for the BK rule for various materials and heavy charged particles. The $\alpha$ values generally decreased with increasing material mass density and atomic number, 
regardless of the ion type, while the $p$ values were weakly dependent on the ion type and target material.

The upper portion of table 3 lists the measured WET values that were measured at the MPRI, and the corresponding calculations based on the BK rule and the NM for various materials. The differences between the values predicted by the BK rule and the NM calculations were less than $0.1 \mathrm{~mm}$, and the differences between results predicted by the BK rule and measured data were less than $0.7 \mathrm{~mm}$. Measurements with proton beams of various cross-sectional areas confirmed that the proton beam range determinations were not influenced by lateral field-size effects. The lower portion of table 3 lists the measured WET values from the NPTC and the corresponding calculations. Differences between the values of WET from the NM calculations and those predicted by the BK rule were less than $0.01 \mathrm{~mm}$, and the differences between the values predicted by the BK rule and the values from the measured data were less than $0.3 \mathrm{~mm}$.

Tables 4-6 list WER predictions from the three analytical formulas and the NM for helium, carbon and iron ions in a variety of materials. Compared to the NM as the standard, the BK rule yielded more accurate estimates of WER than the other analytical formulas for most materials and projectile types and energies. For iron ions, the calculated results from three analytical formulas were comparable to one another. The EBB formula yielded WER predictions that were in better agreement with the NM predictions for all ions and absorber materials considered, except for aluminum, where the differences were negligible. This result is consistent with previous comparisons for protons (Zhang and Newhauser 2009). The reason for the superiority of the EBB approach compared to BB is attributable to the empirical approach to estimate the $I$ value as presented in section 2.1. The values from tables 4-6 also show that helium and heavier projectiles behave in a qualitatively manner similar to those for protons, i.e. the WER values depend on the target material and the beam energy, and the largest deviations from NM occurred when the beam was at the lowest initial energy (Zhang and Newhauser 2009).

It is important to quantify the worst-case errors in WET predictions that were caused by the approximations inherent to the analytical formulas. Figure 2 plots the maximum WET deviations $\left(\Delta R_{\max }\right)$ for different targets and ion species. More precisely, for a given ion type and target material, $\Delta R_{\max }$ is the absolute value of the difference between the WET value from an analytical prediction and that from the corresponding numerical prediction, or $\Delta R_{\max }=\mid$ WET - WET $_{\mathrm{NM} \mid \max }$. The range deviations were all less than $1 \mathrm{~mm}$ regardless of the target material and ion type, except for the lead target in the helium ion beam, which had range deviations as large as $4.08 \mathrm{~mm}$. In this case, restricting the comparison to $119 \mathrm{MeV} / \mathrm{u}(\mathrm{MeV}$ per nucleon) and above initial helium ion energy yielded $\Delta R_{\max }$ values below $1 \mathrm{~mm}$ using the $\mathrm{BK}$ rule, while the $\mathrm{BB}$ and $\mathrm{EBB}$ equations still produced $\Delta R_{\max }$ values larger than $1 \mathrm{~mm}$. However, with a slight modification (i.e. setting $I=13 Z$ for $Z>13$ ), the EBB equation also yielded $\Delta R_{\max }$ values of less than $0.55 \mathrm{~mm}$. Overall, the predictions of WET based on analytical formulas agreed well with the corresponding the NM calculations.

Table 7 lists calculated WER values using the BK rule for proton, carbon ion and iron ion beams and the corresponding measured values from Moyers et al (2009). The data were listed for combinations of different materials, beam types and beam energies. The table also lists the difference between the measured and predicted WET values (WER values were converted to WET values by multiplying the corresponding material thicknesses), denoted by $\Delta R_{\mathrm{BK} \text {,exp }}$. Most of the $\Delta R_{\mathrm{BK} \text {,exp }}$ values were within $1 \mathrm{~mm}$ and all were less than $2.4 \mathrm{~mm}$, and the worst agreement was found for thick targets (thicknesses of 96.5 mm PMMA, $102.9 \mathrm{~mm}$ HDPE, 60.07 and $90.14 \mathrm{~mm}$ bone substitute slabs, $60.37 \mathrm{~mm}$ aluminum). 


\section{Discussion}

In this study, WET values of various materials in heavy charged particle beams calculated by one NM and three analytical formulas were validated against measurements. The good agreement between all measured and predicted values (deviations within $1 \mathrm{~mm}$ in most cases) provides strong evidence that the analytical methods developed in our previous studies predict WET values with adequate accuracy for various clinically relevant ion species, ion energies, and absorber materials.

The International Atomic Energy Agency (IAEA) has proposed techniques to predict the approximate WET of plastic phantoms used for relative proton dosimetry using the ratio of the continuous-slowing-down approximation (CSDA) range (in $\mathrm{g} \mathrm{cm}^{-2}$ ) in water to that in the material of interest and to predict the approximate WET of plastic phantoms used in heavyion beam dosimetry (atomic numbers between 2 and 18 ) as the product of the phantom thickness and the mass density of the phantom (IAEA 2000). Because the CSDA range represents complete loss of ion energy, meaning that the target thickness must be larger than the beam range, this approximation is not applicable in many clinical situations where WET values are needed. Our finding suggests that the simple analytical WET formulas from Zhang and Newhauser (2009), which were developed for protons passing through absorbers of arbitrary thickness and composition, also provide more accurate predictions for helium, carbon and iron ions than those that can be achieved using the IAEA method. The main differences are as follows: the analytical formulas can be used for thick targets and the calculation uncertainties and the interval of applicable energies are now known.

The results of this study have several applications. The analytical formulas used here may be useful in configuring treatment planning systems rather than complex numerical methods; the thicknesses of several radiologically thick components in the treatment head are specified in the planning system in terms of WET (Newhauser et al 2007c, Kramer et al 2000). The analytical formulas may also be particularly useful in calculating WET values for metal implants in patients (Newhauser et al 2007a, 2007b, 2008). Our results can also be used in heavy charged particle beam dosimetry because the absorbed dose measurements depend on the geometric relationships between the particle beam and equipment in the treatment head, and their geometric dimensions are often specified as WET (Newhauser 2001a, 2001b, Newhauser et al 2002, IAEA 2000). While our methods are adequate for many clinical calculations, the requirements on the accuracy of WET predictions depend on many factors, including the treatment site, treatment technique and the proximity of nearby critical structures.

This study had several limitations. First, we neglected density and shell corrections in the BB equation for heavy charged particles following the methods of the previous study on proton WET calculations (Zhang and Newhauser 2009). The calculated results in this paper show that this approximation still yields good accuracy in clinical environments and therefore it is not a serious limitation. Second, the EBB equation required modification of the empirical equation used to calculate $I$ so that errors were less than $1 \mathrm{~mm}$ for the case of the lead target in the helium ion beam. Because this was a rare occurrence, the analytical formula is still applicable in most cases. Third, we did not verify the thin-target approach for heavy charged particle beams other than proton beams (the thin-target approach was tested for the proton beam in our previous study (Zhang and Newhauser 2009)).

In conclusion, we have confirmed that analytical formulas of thick-target approach can be used to calculate WET values of various types of materials in beams of protons, helium, carbon and iron ions with high accuracy. This finding is important because of the increasing use of heavy charged particle beams for cancer treatment and the need for accurate predictions of WET values for such therapies. 


\section{Acknowledgments}

We thank Brian Allen and Draik Hecksel at the MPRI for their assistance with the proton WET measurements, Dr Michael Moyers for the helpful discussions, Dr Uwe Titt for providing lung tissue equivalent material and helpful discussions, and Kathryn B Carnes for her assistance in preparing this manuscript. This work was supported in part by Northern Illinois University through a subcontract of a Department of Defense contract (award W81XWH-08-1-0205) and by the National Cancer Institute (award 1R01 CA131463-01A1).

\section{References}

Amaldi U, Kraft G. Radiotherapy with beams of carbon ions. Rep. Prog. Phys 2005;68:1861-1882.

Anferov, V.; Collins, J.; Friesel, DL.; Katuin, J.; Klein, SB.; Nichiporov, D.; Wedekind, MW. The Indiana University proton therapy system; Proc. of EPAC (Edinburgh, Scotland) WEPCH179; 2006. p. 2349-2351.http://accelconf.web.cern.ch/AccelConf/e06/

Bethe H. Zur Theorie des Durchgangs schneller Korpuskularstrahlen durch Materie. Ann. Physik 1930;5:324-400.

Bloch F. Zur Bremsung rasch bewegter Teilchen beim Durchgang durch Materie. Ann. Physik 1933;16:285-320.

Bragg WH, Kleemann R. On the alpha particles of radium, and their loss of range in passing through various atoms and molecules. Philos. Mag 1905 S.6:318-340.

Goitein M, Lomax A, Pedroni E. Treating cancer with protons. Phys. Today 2002;55:45-50.

IAEA. IAEA Technical Report Series. Vienna: International Atomic Energy Agency; 2000. Absorbed dose determination in external beam radiotherapy.

Jakel O, Schulz-Ertner D, Karger CP, Nikoghosyan A, Debus J. Heavy ion therapy: status and perspectives. Technol. Cancer Res. Treat 2003;2:377-387. [PubMed: 14529303]

Jongen Y, Beeckman W, Cohilis P. The proton therapy system for MGH's NPTC: equipment description and progress report. Bull. Cancer Radiother 1996;83:219s-222s. [PubMed: 8949784]

Kramer M, Jakel O, Haberer T, Kraft G, Schardt D, Weber U. Treatment planning for heavy-ion radiotherapy: physical beam model and dose optimization. Phys. Med. Biol 2000;45:3299-3317. [PubMed: 11098905]

Leo, WR. Techniques for Nuclear and Particle Physics Experiments. Berlin: Springer; 1987.

Moyers MF, Sardesai M, Sun S, Miller DW. Ion stopping powers and CT numbers. Med. Dosim. 2009 at press.

Newhauser, W. Dosimetry for the gantry beams at the northeast proton therapy center: part I. Dimensions and geometric relationships. Massachusetts General Hospital Report HD-112. 2001a.

Newhauser, W. Proton field calibration measurements and analysis with the calibration setup program (CSU) version 1.1. Massachusetts General Hospital Report. 2001b.

Newhauser W, Fontenot J, Koch N, Dong L, Lee A, Zheng Y, Waters L, Mohan R. Monte Carlo simulations of the dosimetric impact of radiopaque fiducial markers for proton radiotherapy of the prostate. Phys. Med. Biol 2007a;52:2937-2952. [PubMed: 17505081]

Newhauser W, Fontenot J, Zheng Y, Polf J, Titt U, Koch N, Zhang X, Mohan R. Monte Carlo simulations for configuring and testing an analytical proton dose-calculation algorithm. Phys. Med. Biol 2007c; 52:4569-4584. [PubMed: 17634651]

Newhauser W, Myers KD, Rosenthal SJ, Smith AR. Proton beam dosimetry for radiosurgery: implementation of the ICRU Report 59 at the Harvard Cyclotron Laboratory. Phys. Med. Biol 2002;47:1369-1389. [PubMed: 12030561]

Newhauser WD, Giebeler A, Langen KM, Mirkovic D, Mohan R. Can megavoltage computed tomography reduce proton range uncertainties in treatment plans for patients with large metal implants? Phys. Med. Biol 2008;53:2327-2344. [PubMed: 18421122]

Newhauser WD, Koch NC, Fontenot JD, Rosenthal SJ, Gombos DS, Fitzek MM, Mohan R. Dosimetric impact of tantalum markers used in the treatment of uveal melanoma with proton beam therapy. Phys. Med. Biol 2007b;52:3979-3990. [PubMed: 17664589]

Schulz-Ertner D, Jakel O, Schlegel W. Radiation therapy with charged particles. Semin. Radiat. Oncol 2006;16:249-259. [PubMed: 17010908] 
Schulz-Ertner D, Tsujii H. Particle radiation therapy using proton and heavier ion beams. J. Clin. Oncol 2007;25:953-964. [PubMed: 17350944]

Wilson RR. Radiological use of fast protons. Radiology 1946;47:487-491. [PubMed: 20274616]

Zhang R, Newhauser WD. Calculation of water equivalence of materials of arbitrary density, elemental composition and thickness in proton beam irradiation. Phys. Med. Biol 2009;54:1383-1395. [PubMed: 19218739]

Ziegler, JF.; Biersack, JP.; Littmark, U. The Stopping and Ranges of Ions in Solids. New York: Pergamon; 1985. 


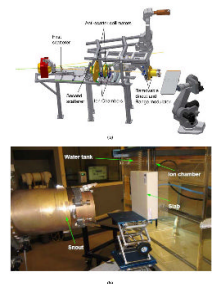

Figure 1.

(a) Schematic diagram of the fixed horizontal proton beam line configuration at the MPRI (Courtesy of Indiana University Cyclotron Facility). (b) Experimental apparatus at the end of the proton beam line at the MPRI for measuring the WET of one material (a lung substitute plastic slab). 


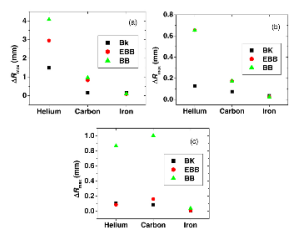

Figure 2.

Maximum deviations, $\Delta R_{\max }$, in WET values calculated by the analytical formulas relative to that given by the NM for (a) lead, (b) aluminum and (c) PMMA. The thicknesses of materials used were $1.5 \mathrm{~cm}$ lead, $2 \mathrm{~cm}$ aluminum and $10 \mathrm{~cm}$ PMMA for helium ions; $1.2 \mathrm{~cm}$ lead, 1.5 $\mathrm{cm}$ aluminum and $10 \mathrm{~cm}$ PMMA for carbon ions; $0.1 \mathrm{~cm}$ lead, $0.2 \mathrm{~cm}$ aluminum and $0.3 \mathrm{~cm}$ PMMA for iron ions. 
Table 1

Common materials used in heavy charged particle beams, with their mass densities $\rho$, values of $(Z / A)_{\text {eff }}$ and mole fractions.

\begin{tabular}{|c|c|c|c|}
\hline Material & $\rho\left(\mathrm{g} \mathrm{cm}^{-3}\right)$ & $(Z / A)_{\text {eff }}$ & Mole fraction (\%) \\
\hline Lung substitute & 0.3 & 0.537 & $\begin{array}{l}\text { H 55.577, C 32.738, N 0.927, O 7.508, } \\
\text { Cl } 0.019, \text { Si } 0.184, \mathrm{Mg} 3.048\end{array}$ \\
\hline HDPE & 0.964 & 0.570 & H 66.717, C 33.283 \\
\hline Water & 1.0 & 0.555 & Н 66.667, О 33.333 \\
\hline Polystyrene & 1.06 & 0.538 & Н 49.851, С 50.149 \\
\hline PMMA & 1.185 & 0.539 & Н 53.333, С 33.333 , O 13.333, \\
\hline Lexan & 1.20 & 0.527 & H 42.424, C 48.485, O 9.091 \\
\hline PVC & 1.385 & 0.512 & H 50.218, C 33.202, Cl 16.580 \\
\hline Bone substitute & 1.829 & 0.516 & H 35.215, C 29.592, N 0.803, O 26.695, Cl 0.16, Ca 7.679 \\
\hline Aluminum & 2.698 & 0.482 & Al 100 \\
\hline Titanium & 4.519 & 0.459 & Ti 100 \\
\hline Stainless steel & 7.85 & 0.466 & $\begin{array}{l}\text { C 0.045, N 0.045, Si 0.450, Cr 18.150, Mn 1.250, } \\
\text { Fe 71.460, Ni 8.550, Mo } 0.050\end{array}$ \\
\hline Lead & 11.322 & 0.396 & $\mathrm{~Pb} 100$ \\
\hline Gold & 19.311 & 0.401 & $\mathrm{Au} 100$ \\
\hline
\end{tabular}




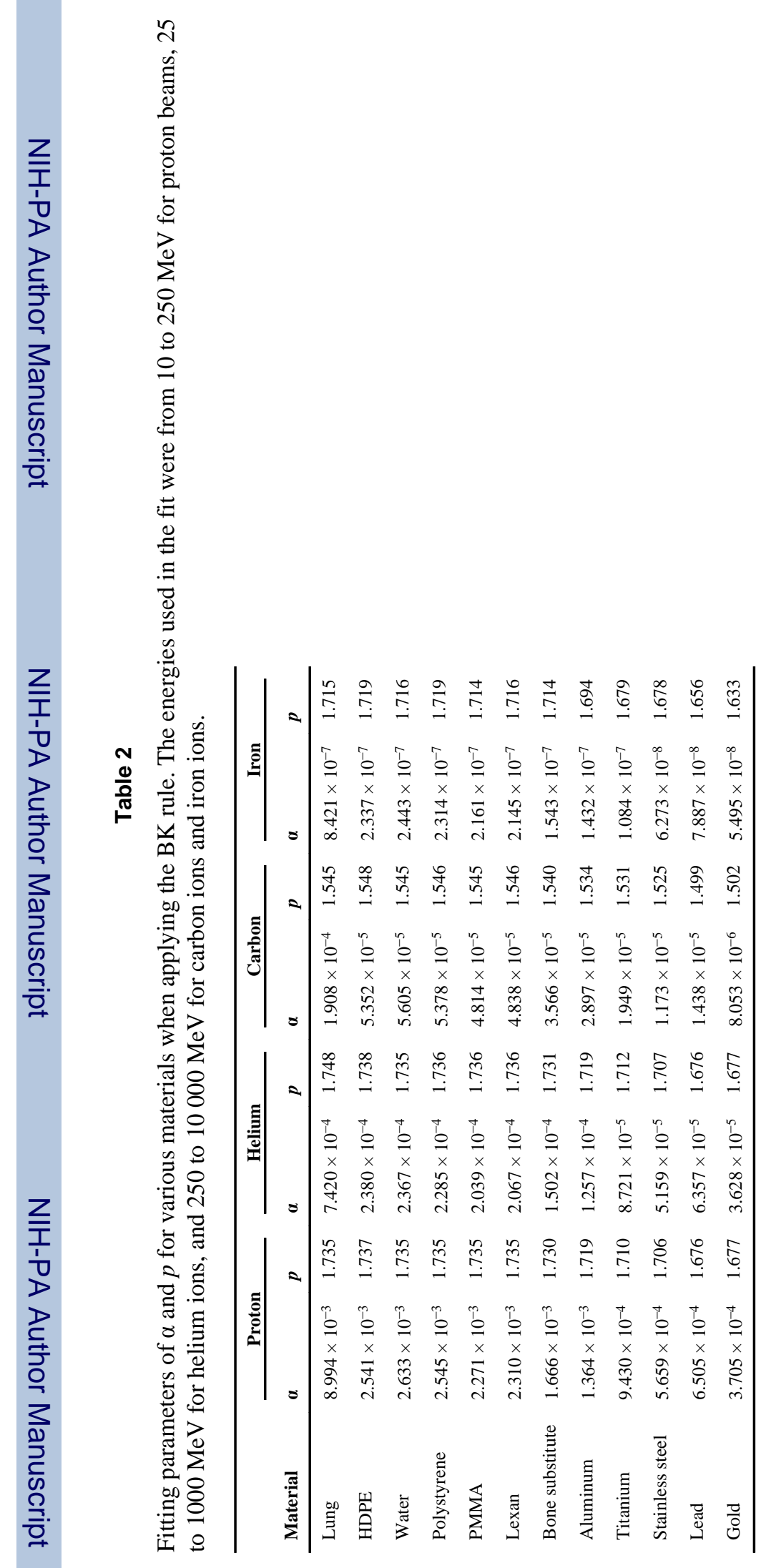

Phys Med Biol. Author manuscript; available in PMC 2010 November 10. 


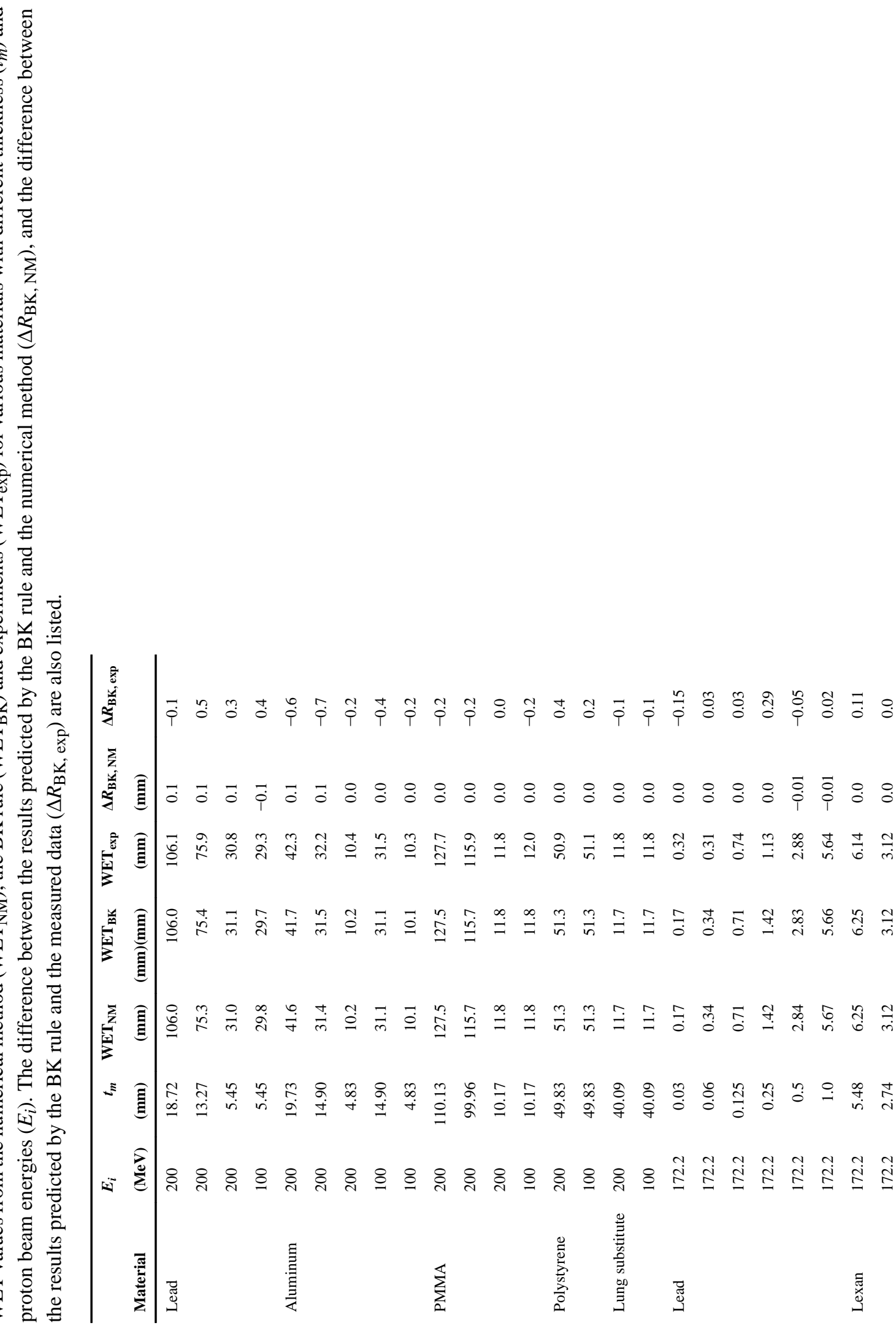




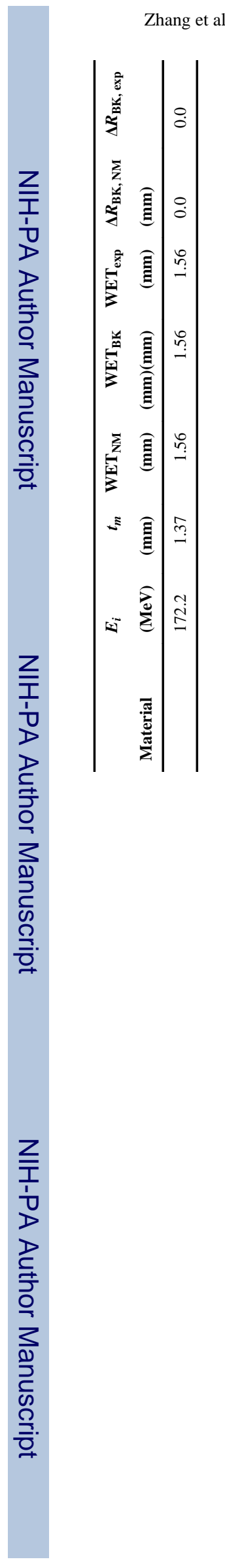

Page 14 


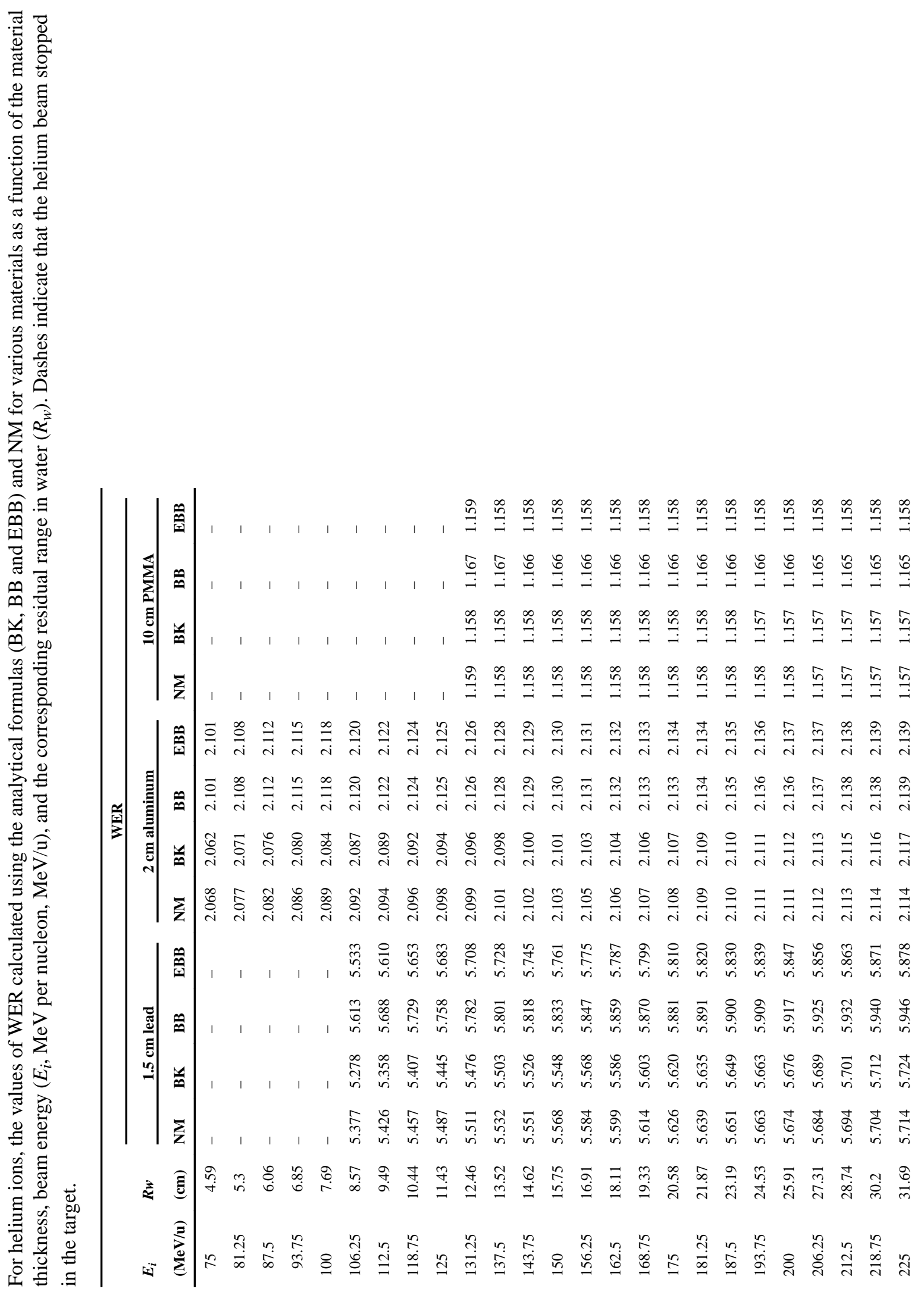




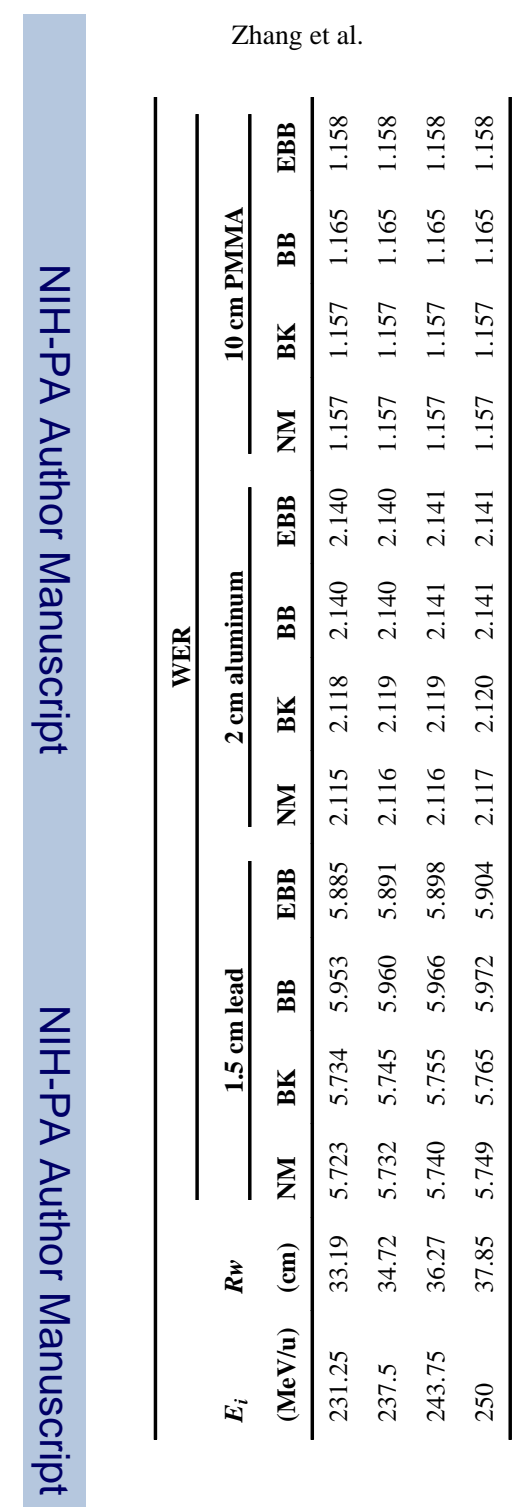

Page 16 


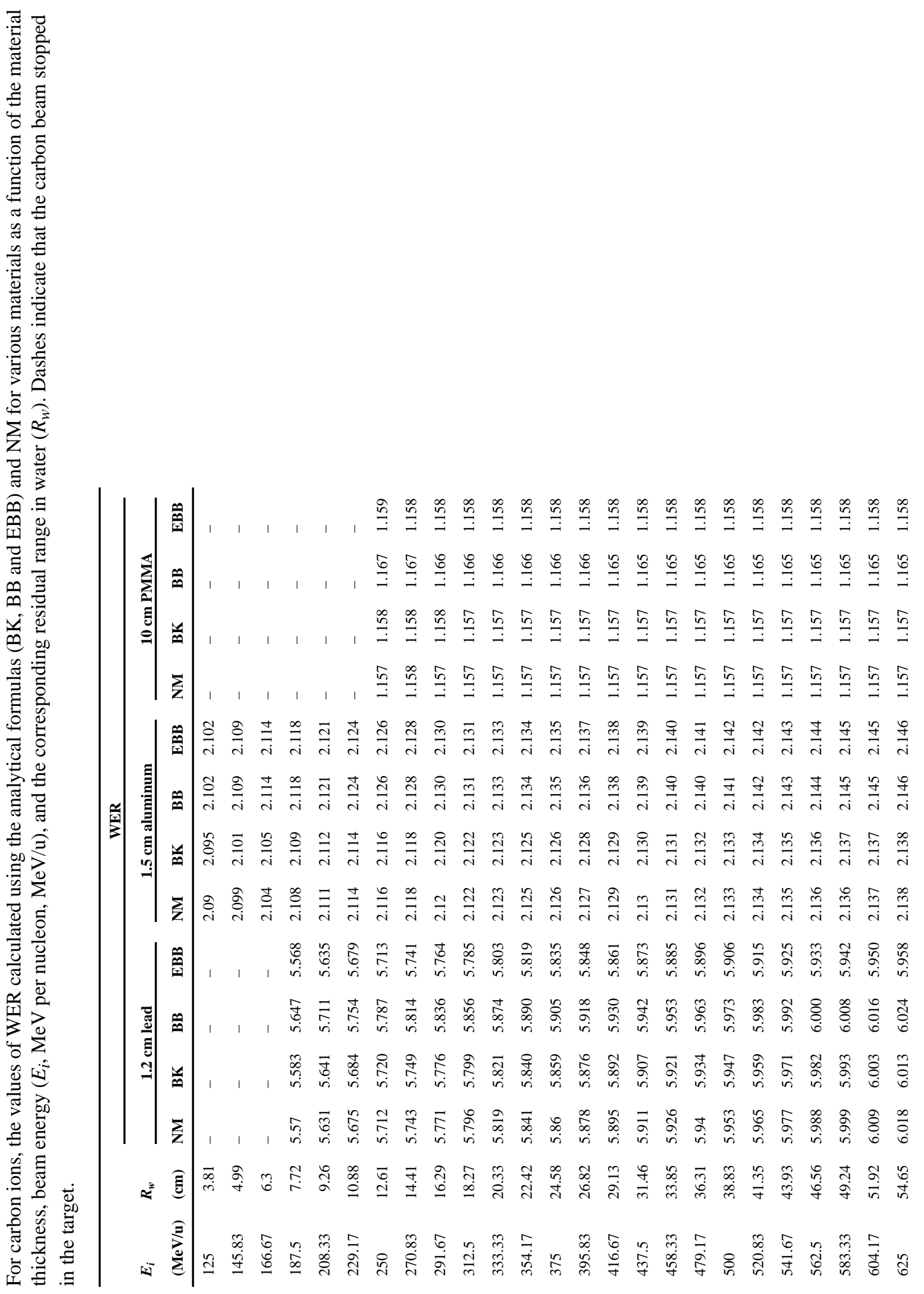




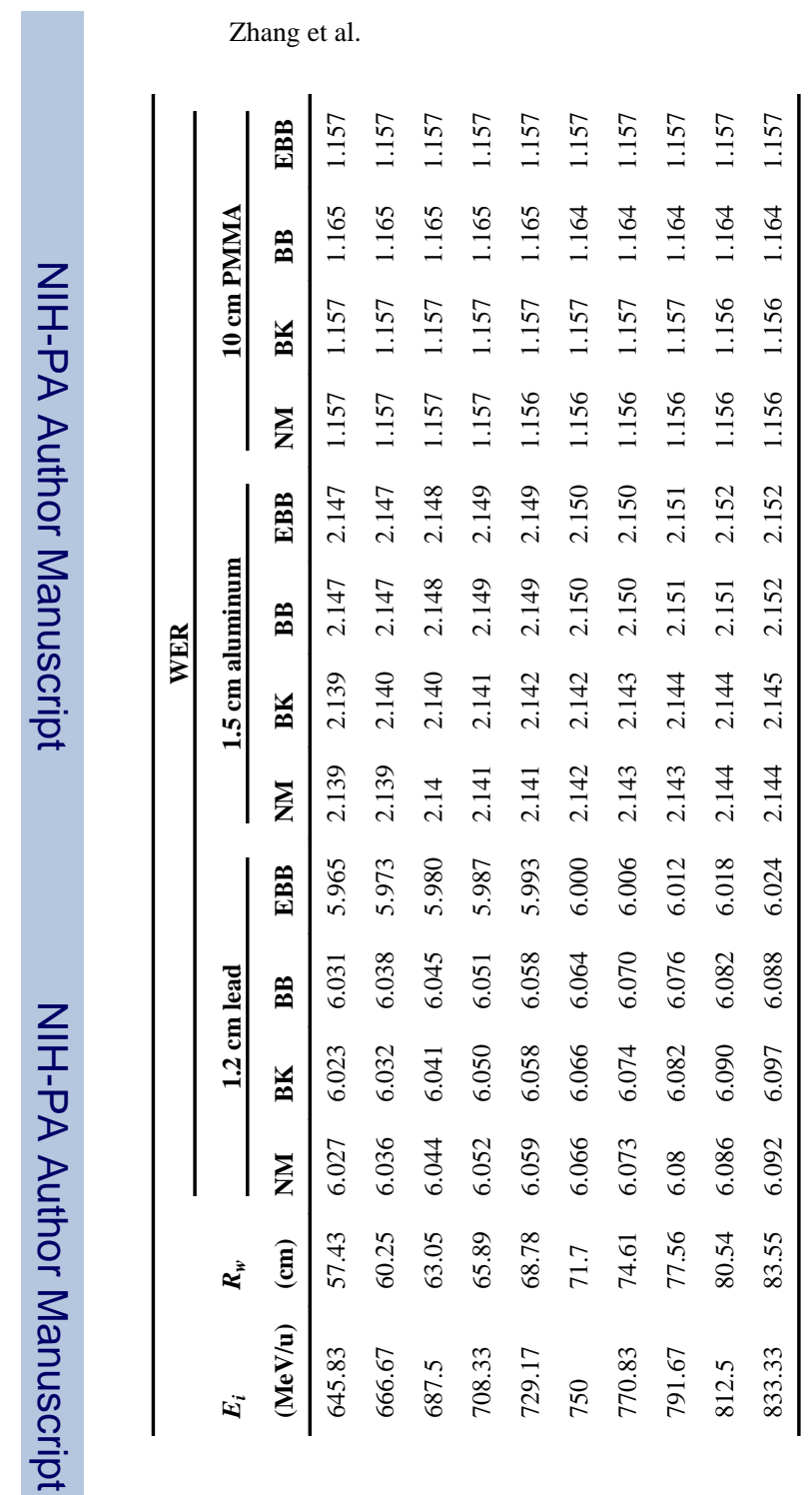




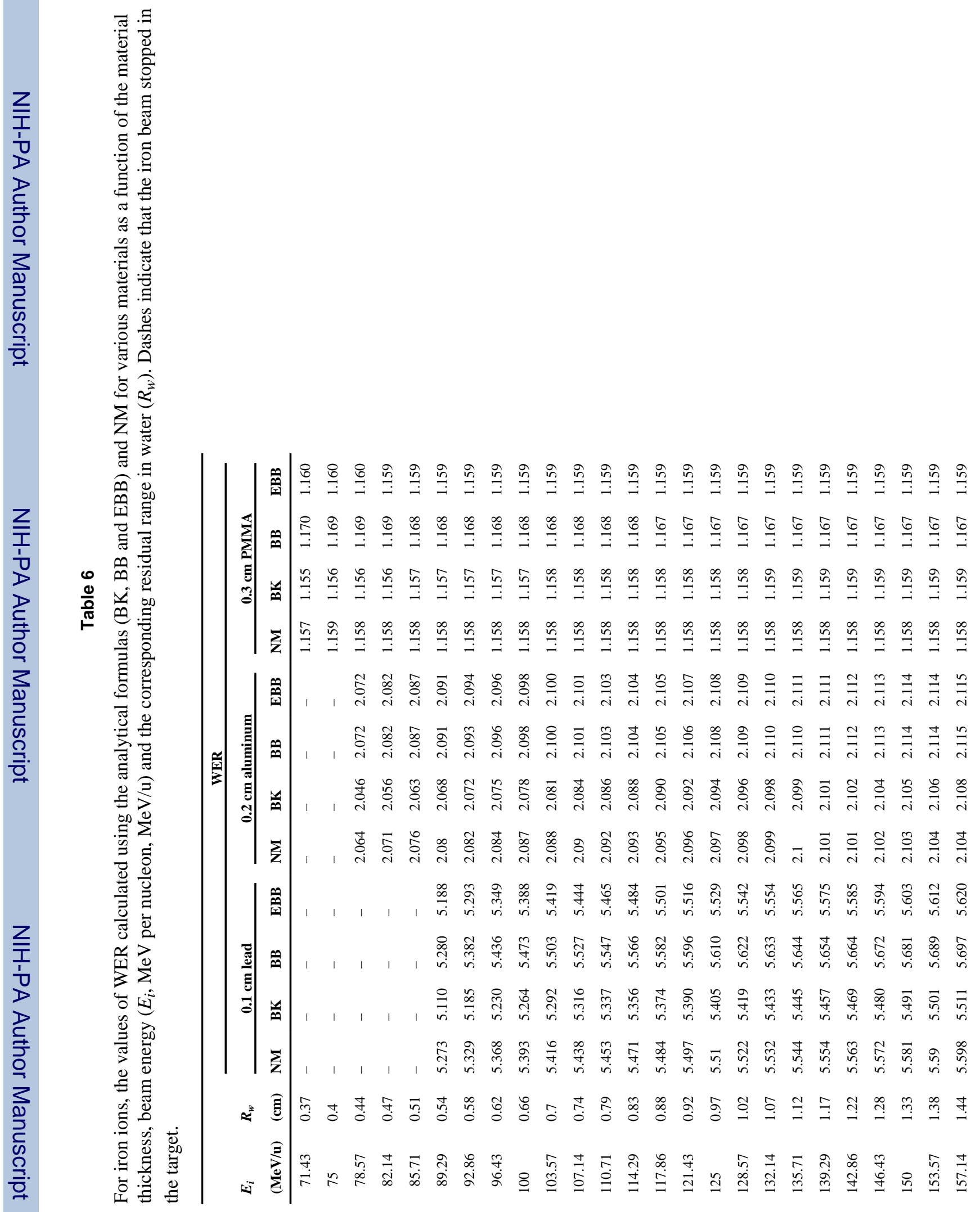




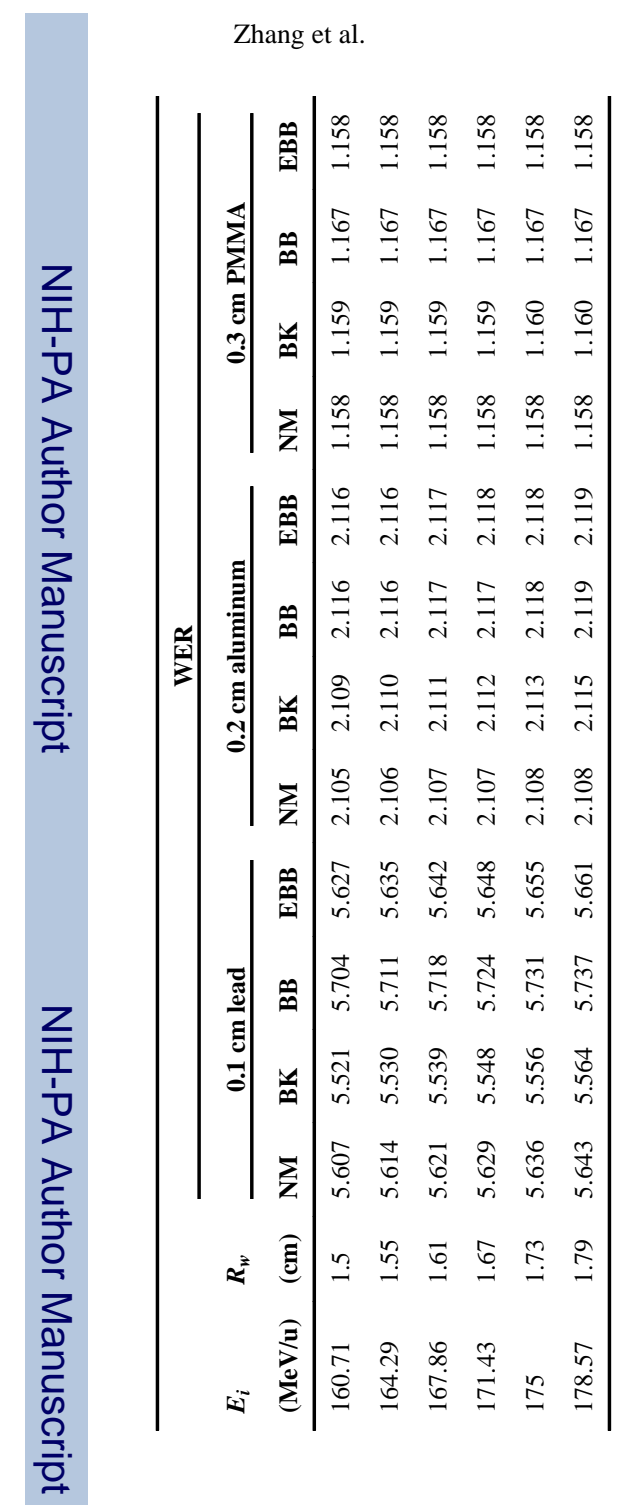


ถี 